\title{
THE KINETIC METHOD IN STATISTICAL MECHANICS.
}

$\mathrm{BY}$

G. E. UHLENBECK, PH.D.,

University of Michigan.

So much has been said to-day about the statistical interpretation of quantum-mechanics, that perhaps one should like to see now quite clearly, how the fundamental postulates of the ordinary kinetic theory and statistical mechanics, ${ }^{1}$ especially their connection with thermodynamics, are "explained" by the quantum theory. In principle this is nowadays certainly possible, ${ }^{2}$ I think, though I am not able to show you exactly how. Therefore only a few remarks, especially on the so-called kinetic method.

We can distinguish in the kinetic theory two fundamental methods:

a. The statistical method; the equilibrium state is described as the most probable or as the mean state. The range of application is about the same as of ordinary thermodynamics, ${ }^{3}$ because it deals principally with equilibrium states, with the exception ${ }^{4}$ that it can explain also the fuctuation-phenomena around an equilibrium state.

$b$. The kinetic method or method of collisions. Here we try to explain the macroscopic properties by considering more precisely the interaction between the particles (atoms, light quanta, etc.), out of which our system is composed. It is the only method, for systems not in an equilibrium state, for finding the rate of physical and chemical processes. Especially it gives, in principle, by means of the $H$-theorem of Boltzmann, something about the velocity with which the equilibrium state is reached. A disadvantage is, that it

${ }^{1}$ Compare for instance my dissertation, Over statistische methoden in de theorie der quanta (The Hague, Nyhoff, 1927).

${ }^{2}$ Compare J. v. Neumann, Gött. Nachr., 1927, p. 273.

${ }^{3}$ Partly, it is simply another language.

${ }^{4}$ This is, perhaps, not exactly true, in the sense that by a slight reformulation of the second law of thermodynamics one can also account for the fluctuationphenomena. Compare L. Szilard, ZS. f. Phys., v. 32, p. 753, 1925. 
cannot account for the fluctuation-phenomena, at least not in a general way.

I think that quantum-mechanics will combine these two, now almost distinct, methods by sharpening the kinetic method, using statistical considerations.

In the kinetic method we can deduce, at least formally, all the thermodynamic laws by the following assumptions: ${ }^{5}$

I. The "Stoszzahlansatz": the number of collisions per sec. of particles in the quantum state $i$ with particles in the state $j$, where $k$ and $l$ are the states after collision, is:

$$
A_{i j}{ }^{k l}=a_{i j}{ }^{k l} n_{i} n_{j}\left(\mathbf{I}+\theta n_{k}\right)\left(\mathrm{I}+\theta n_{l}\right) .
$$

$a_{i j}{ }^{k l}$ is a sort of transition probability, and

$$
\begin{aligned}
& \theta=-\mathrm{I} \text { for a Fermi-Dirac-gas, } \\
& \theta=\mathrm{o} \text { in the classical case, } \\
& \theta=+\mathrm{I} \text { for a Bose-Einstein-gas. }
\end{aligned}
$$

2. The principle of microscopic reversibility: ${ }^{6}$

$$
a_{i j}{ }^{k l}=a_{k l}{ }^{i j} \text {. }
$$

Introducing a generalized $H$-function of Boltzmann by ${ }^{7}$

$$
H=\sum_{i}\left\{n_{i} \log n_{i}-\theta\left(\mathrm{I}+\theta n_{i}\right) \log \left(\mathrm{I}+\theta n_{i}\right)\right\},
$$

we can show that $(d H / d t)<0$ until the equilibrium-state is reached, which is determined by the functional equation

$$
\bar{n}_{i} n_{j}\left(\mathrm{I}+\theta n_{k}\right)\left(\mathrm{I}+\theta \bar{n}_{l}\right)=\bar{n}_{k} n_{l}\left(\mathrm{I}+\theta \bar{n}_{i}\right)\left(\mathrm{I}+\theta \bar{n}_{j}\right) \text {, }
$$

where the bar denotes the values in the equilibrium state. From this follows immediately, when we take into account only the conservation of energy during each collision (no translational motion of the whole gas):

$$
\log \frac{n_{i}}{\mathrm{I}+\theta n_{i}}=-\beta \epsilon_{i}+\log A
$$

${ }_{5}^{5}$ This is for a single gas. Compare P. Jordan, ZS. f. Phys., v. 4r, p. 7 I I, 1927; L. S. Ornstein and H. A. Kramers, ZS. f. Phys., v. 42, p. 48r, I927; L. Nordheim, Proc. Roy. Soc., series A., v. 119, p. 689, 1928.

${ }^{B}$ See the discussion in R. C. Tolman, Statistical Mechanics (New York, The Chemical Catalog Company, 1927), chapter I 5.

7 The entropy is always given by $S=-k H$. 
or

$$
n=\frac{\mathrm{I}}{\frac{\mathrm{I}}{A} e^{\beta \varepsilon i}-\theta} .
$$

It is easy to generalize this for mixtures, to find the analogue of the transport equation of Boltzmann, and so on. ${ }^{8}$

I wanted further only to point out that, in the case of the interaction of light and fixed atoms, all these formal assumptions are beautifully explained by the theory of Dirac. $^{9} \quad$ Especially the principle of microscopic reversibility is there "proved" by showing that $a_{i j}{ }^{k l}$ is equal to the square of the absolute value of an Hermitean matrix.

With the help of this we can see also more precisely how fast the equilibrium state (Planck's radiation formula) is established. As is well known, Planck always mentioned that we need "a little piece of coal dust" to establish the equilibrium, when we have the radiation in a vessel, of which the walls are perfect mirrors. We may ask now, how big this piece of coal dust has to be?

First it is clear that we need some matter, because light waves or light quanta have no way to interact, will therefore not "collide," or, we may say, are perfectly penetrable for each other. Let us therefore take first one free electron. One sees easily that, when we consider only the Comptonscattering, this will not establish the equilibrium. ${ }^{10}$ Because then, also, the number of light quanta is conserved (besides their energy), and from the statistical treatment it follows that we can only get

$$
\bar{n}_{i}=\frac{\mathrm{I}}{\frac{\mathrm{I}}{A} e^{\beta e i}-\mathrm{I}}
$$

and not Planck's formula, for which $A=\mathrm{I}$. We have to

${ }^{8}$ See L. Nordheim, Proc. Roy. Soc., series A., v. I I9, p. 689, 1928.

${ }^{9}$ P. A. M. Dirac, Proc. Roy. Soc., v. I14, p. 243, 1927; v. II4, p. 710, I927. Compare also the treatment in H. Weyl: Gruppentheorie und Quantenmechanik (Leipzig, S. Hirzel, 1928), $\$ 20$ and 44 . For the extension to an electron gas, see esp. P. Jordan and E. Wigner, ZS. f. Phys., v. 47, p. 63 I, 1928.

${ }^{10}$ P. Jordan, ZS. f. Phys., v. 33, p. 649, 1925. W. Bothe, ZS. f. Phys., v. 23, p. 2I4, I924. 
determine $A$ from the condition that

$$
\sum_{i} \bar{n}_{i}=N .
$$

The function $N(A)$ will be zero for $A=0$, and infinite for $A=\mathrm{I}$, and is always curved toward the $A$ axis. It is clear, that the electron will very slowly make $N$ infinite, namely by triple and higher collisions. It is therefore not a very effective "piece of coal dust." Neither is one Hydrogenatom, as we see from Dirac's treatment, because that can only establish Planck's law for its own discrete frequencies; at least its effectiveness for other frequencies must be very slight, since here again collisions of higher order are necessary. So that we may say, perhaps, that we really need a piece of crystal, or at least some material system with a practically continuous energy spectrum, to establish the equilibrium rapidly.

In conclusion, I may say, perhaps, that it seems to me better now to stress more the difference between light quanta and electrons, than their similarity. I have the impression that light is still more "wavy" than electrons, for the following reasons:

$a$. For light quanta holds the Bose-Einstein statistics which is equivalent to quantized eigenvibrations; for electrons we have the Fermi-Dirac statistics, which cannot be translated in a natural way into a three-dimensional wave language.

$b$. To find the coupling terms between light and matter we use always the classical expressions following from the electro-magnetic wave-theory of light and from the corpuscular theory of matter (esp. Coulomb's law).

Department of Physics, University of Michigan. 